\title{
Histopathological Characterization of Cases of Spontaneous Fatal Feline Severe Fever with Thrombocytopenia Syndrome, Japan
}

\author{
Yusuke Sakai, Yuko Kuwabara, Keita Ishijima, ${ }^{1}$ Saya Kagimoto, Serina Mura, \\ Kango Tatemoto, ${ }^{1}$ Ryusei Kuwata, ${ }^{2}$ Kenzo Yonemitsu, ${ }^{1}$ Shohei Minami, Yudai Kuroda, ${ }^{1}$ \\ Kenji Baba, Masaru Okuda, Hiroshi Shimoda, Masashi Sakurai, Masahiro Morimoto, Ken Maeda ${ }^{1}$
}

\begin{abstract}
Severe fever with thrombocytopenia syndrome (SFTS) is an emerging tickborne infectious disease caused by SFTS virus (SFTSV). We report 7 cases of spontaneous fatal SFTS in felines. Necropsies revealed characteristic lesions, including necrotizing lymphadenitis in 5 cases and necrotizing splenitis and SFTSV-positive blastic lymphocytes in all cases. We detected hemorrhagic lesions in the gastrointestinal tract in 6 cases and lungs in 3 cases, suggesting a more severe clinical course of SFTS in felids than in humans. We noted necrotic or ulcerative foci in the gastrointestinal tract in 3 cases, the lung in 2 cases, and the liver in 4 cases. We clarified that blastic lymphocytes are predominant targets of SFTSV and involved in induction of necrotic foci. We also found that thymic epithelial cells were additional targets of SFTSV. These results provide insights for diagnosing feline SFTS during pathological examination and demonstrate the similarity of feline and human SFTS cases.
\end{abstract}

Cevere fever with thrombocytopenia syndrome $\mathcal{S}$ (SFTS) is an emerging infectious disease characterized by acute onset of high fever, hemorrhagic tendency, gastrointestinal and neurologic symptoms, thrombocytopenia, and leukocytopenia (1-4). The causative agent of SFTS is a novel Dabie bandavirus, SFTS virus (SFTSV), of the family Phenuiviridae (5). On the basis of epidemiologic evidence, SFTS is classified as a tickborne disease, and the main reservoir and vector involving human infection is thought to be the Haemaphysalis longicornis tick $(6,7)$. In addition, various species of domestic and wild mammals, including goats, sheep, cattle, pigs, dogs, cats, boars, and deer, have been found to harbor SFTSV genomic RNA or SFTSV antibodies (8-10).

Authors affiliation: Yamaguchi University, Yamaguchi, Japan

DOI: https://doi.org/10.3201/eid2704.204148
These data demonstrate the infectivity of SFTSV in these animal species and circulation of SFTSV between ticks and animals in nature. Reported cases of SFTS in cheetahs and domestic cats have shown that nonhuman mammalian species can develop fatal disease similar to human SFTS $(11,12)$. Susceptibility of cats to SFTSV also was confirmed by experimental SFTSV infection in cats, which caused a high incidence of severe hemorrhagic fever (13). Analyses of these cases confirmed shedding of viral particles from saliva and feces $(11,13)$, which can cause transmission of SFTSV from diseased animals to humans (14).

Analysis of animals with SFTSV infection can inform the pathogenesis of SFTS in humans. Experimental infection in wild type and $\alpha / \beta$ interferon receptor knockout mice, rhesus macaques (Macaca mulatta), and signal transducer and activator of transcription-2 knockout hamsters have been reported (15-19), but hepatitis and splenitis have been reproduced only in hamster models (19). However, hemorrhagic and necrotic lesions in the liver, spleen, intestines, and lymph nodes have been reported only in fatal SFTS cases in humans and experimentally infected felines $(13,20-22)$. Investigations of disease in animals that mimics human SFTS is crucial for informing prevention of animal-to-human transmission and controlling virus transmission among animals and ticks. Analysis of fatal cases in felines can clarify the pathology of SFTS and inform STFS diagnosis in animals. We provide evidence of characteristic macroscopic and microscopic lesions collected from 7 cases of spontaneous fatal SFTS in felines.

${ }^{1}$ Current affiliation: National Institute of Infectious Diseases, Tokyo, Japan.

${ }^{2}$ Current affiliation: Okayama University of Science, Ehime, Japan. 


\section{Materials and Methods}

\section{Histology}

We performed necropsies on 7 cats with SFTS symptoms, such as acute onset of thrombocytopenia, leukocytopenia, and lethargy (Table 1). We confirmed SFTSV infection by conventional reverse transcription PCR (RT-PCR) using 2 primer pairs targeting the small segment of the SFTSV genome (23). We collected and fixed tissue samples in 10\% neutral buffered formalin and then processed the samples to create paraffin-embedded tissue sections. We cut tissue into sections $4-\mu \mathrm{m}$ thick and stained sections with hematoxylin and eosin for histopathologic examination.

\section{Immunohistochemistry}

We subjected the 4- $\mu \mathrm{m}$ thick tissue sections to immunohistochemical staining. After deparaffinization, we performed antigen retrieval by incubating sections in $0.1 \%$ trypsin at $37^{\circ} \mathrm{C}$ for $20 \mathrm{~min}$ to obtain immunoglobulin (Ig) lambda chain; or by heating at $121^{\circ} \mathrm{C}$ for $5 \mathrm{~min}$ in $\mathrm{pH} 6.0$ citrate buffer for SFTSV and CD3 staining; or $\mathrm{pH} 9.0$ Tris-EDTA buffer for CD79a and Ki67 staining. After washing with phosphate-buffered saline (PBS), we inactivated endogenous peroxidase by immersion in 3\% hydrogen peroxide in PBS. After treatment with $5 \%$ bovine serum albumin in PBS for $30 \mathrm{~min}$, we incubated the sections with rabbit polyclonal anti-SFTSV antibody (diluted 1:1,000; gift from Shigeru Morikawa, Okayama University of Science, Okayama, Japan); FLEX Polyclonal Rabbit AntiHuman CD3 Ready-to-Use antibody (Dako, https:/ / www.agilent.com); Monoclonal Mouse Anti-Human CD79a Antibody Clone HM57 (diluted 1:50; Dako); Mouse Monoclonal Anti-Ki67 Clone MIB-1 (diluted 1:1,000; eBioscience, https://www.thermofisher. com); or Polyclonal Rabbit Anti-Human Ig Lambda
Light Chains (diluted 1:100; Dako). After washing with PBS, we incubated the sections with EnVision+/ HRP Rabbit (Dako) horseradish peroxidase (HRP)-labeled polymer anti-rabbit or EnVision+/HRP Mouse (Dako) HRP-labeled polymer anti-mouse. We then visualized positive signals by peroxidase-diaminobenzidine reaction, and counterstained sections with hematoxylin stain.

\section{Immunofluorescence}

We performed double immunofluorescence labeling with cytokeratin-SFTSV and CD204-SFTSV on 4- $\mu \mathrm{m}$ thick tissue sections. We performed heat-mediated antigen retrieval for cytokeratin-SFTSV in $\mathrm{pH} 6.0$ citrate buffer and for CD204-SFTSV antigen pH 9.0 Tris-EDTA buffer. After washing with PBS and blocking with $5 \%$ bovine serum albumin, we incubated the sections for $1 \mathrm{~h}$ at room temperature with a mixture of rabbit polyclonal anti-SFTSV antibody (diluted 1:1,000; TransGenic Inc., https://www.transgenic.co.jp) and mouse monoclonal anti-CD204 (diluted 1:400; TransGenic Inc.) or mouse monoclonal anti-cytokeratin clone AE1/AE3 (diluted 1:200; Dako). After washing with PBS, we incubated the sections in a mixture of Alexa Fluor 488 anti-rabbit IgG (diluted 1:400; Abcam, https://www.abcam.com), Alexa Fluor 594 antimouse IgG (diluted 1:400; Abcam), and DAPI (Dojindo, https:/ / www.dojindo.com). We then analyzed the tissue sections by using an LSM 710 (Leica, https:/ / www.leicabiosystems.com) confocal microscope.

\section{Results}

\section{Gross Findings}

Among the 7 cats with SFTS, gross lesions typically were characterized by changes in the lymphoid organs and hemorrhage (Table 2). In all the cases, we

\begin{tabular}{|c|c|c|c|c|c|c|c|}
\hline \multirow[b]{2}{*}{ Clinical findings } & \multicolumn{7}{|c|}{ Case no. } \\
\hline & 1 & 2 & 3 & 4 & 5 & 6 & 7 \\
\hline \multicolumn{8}{|l|}{ Clinical signs } \\
\hline Anorexia & Y & Y & Y & Y & Y & $\mathrm{Y}$ & Y \\
\hline Lethargy & Y & Y & Y & Y & Y & Y & Y \\
\hline Neurologic signs & $\mathrm{N}$ & $\mathrm{N}$ & $\mathrm{N}$ & Y & $\mathrm{N}$ & $\mathrm{N}$ & $\mathrm{N}$ \\
\hline Vomiting & $\mathrm{N}$ & Y & $\mathrm{N}$ & $\mathrm{N}$ & $\mathrm{N}$ & Y & $\mathrm{N}$ \\
\hline Body temperature, ${ }^{\circ} \mathrm{C}$ & 39.4 & NA & NA & NA & 39.3 & 39.9 & 39 \\
\hline Erythrocytes, $10^{4}$ cells $/ \mu \mathrm{L}$ & 546 & 688 & NA & 364 & 718 & 820 & 593 \\
\hline Leukocytes, cells/ $\mu \mathrm{L}$ & 3,080 & 800 & 3,000 & 190 & 700 & 2,500 & 1,290 \\
\hline Platelets, cells $/ \mu \mathrm{L}$ & 38,000 & 7,000 & 0 & 0 & $<11,000$ & 52,000 & 0 \\
\hline $\mathrm{ALT}, \mathrm{IU} / \mathrm{L}$ & 105 & NA & 476 & 141 & 331 & NA & 58 \\
\hline AST, IU/L & 51 & NA & $>1,000$ & 4 & 1,010 & NA & NA \\
\hline ALP, IU/L & 188 & NA & NA & NA & NA & NA & $<10$ \\
\hline Total bilirubin, $\mathrm{mg} / \mathrm{dL}$ & 4.4 & 5.8 & 4.8 & 5.7 & NA & NA & 9.3 \\
\hline CPK, IU/L & NA & 373 & $>2,000$ & $>2,000$ & 1,444 & NA & NA \\
\hline
\end{tabular}

*ALP, alkaline phosphatase; ALT, alanine aminotransferase; AST, aspartate aminotransferase; CPK, creatine phosphokinase; NA, not available. 
noted red enlarged lymph nodes from various regions. Although splenomegaly was unclear to mild, we noticed enlarged follicles appearing as multiple white spots in the spleen (Figure 1, panel A) in all cats. We detected hemorrhagic lesions in the gastrointestinal tract in 6 cats (Figure 1, panel B) and in the tracheal region of the lungs in 3 cats (Figure 1, panel C). In 3 cats, gastrointestinal lesions resulted in grossly obvious ulcers (Figure 1, panel D). Jaundice was detected in 5 cats.

\section{Lymphatic System Lesions}

Histologically, characteristic SFTS lesions were observed in the lymphoid organs, such as the lymph nodes, spleen, and Peyer's patches. We noted lesions in the collected lymph nodes in all 7 cats (Table 3 ). In the cortex of SFTSV-affected lymph nodes, we observed an accumulation of the large blastic lymphocytes, as those described in a human case (21). The blastic lymphocyte cells were morphologically characterized by large, clear irregular-shaped nuclei with prominent central nucleoli and were similar to the morphology of the immature activated-B cells, called immunoblasts (Figure 2, panels A, B). Compatible with immunoblast-like cell morphology, these cells were considered cells of the $\mathrm{B}$ cell lineage because they were positive for CD79a expression (Figure 2, panel C). Immunohistochemistry also revealed that SFTSV antigens were exclusively detected in these blastic lymphocytes and SFTSV-positive blastic lym-
Table 2. Gross lesions in 7 cats with fatal severe fever with thrombocytopenia syndrome, Japan

\begin{tabular}{lc}
\hline Lesions & Cases, no. $(\%)$ \\
\hline Enteric hemorrhage & $6(85.7)$ \\
Gastrointestinal ulcer & $3(42.8)$ \\
Pulmonary hemorrhage & $3(42.8)$ \\
Jaundice & $5(71.4)$ \\
\hline
\end{tabular}

phocytes distributed in the cortex and paracortex area surrounding lymphoid follicles (Figure 2, panels D, E). Regardless of enlargement, some lymph nodes had neither SFTSV-positive cells nor necrotic lesions and were simply diagnosed as hyperplastic lymph nodes (Table 3; Figure 2, panel F). In some lymph nodes, the lesions proceeded to necrotizing lymphadenitis with SFTSV-positive blastic lymphocytes (Figure 2, panel G). In all cases, we detected SFTSV-positive blastic lymphocytes and necrotic foci in the spleen, mainly in the follicular area (Figure 2, panel $\mathrm{H}$ ). We collected the thymus glands from 4 cats and observed infiltration of SFTSV-positive blastic lymphocytes mainly in the cortices of all specimens. Hemorrhagic and necrotic lesions also were observed in the thymus glands. As reported in humans (20-22), we observed numerous hemophagocytic macrophages in the lymphoid organs of all cases.

\section{Intestinal Tract Lesions}

We noted SFTSV-positive blastic lymphocytes in the intestinal tract in all cases (Figure 3), mainly in the Peyer's patches, the localized lymphoid follicular

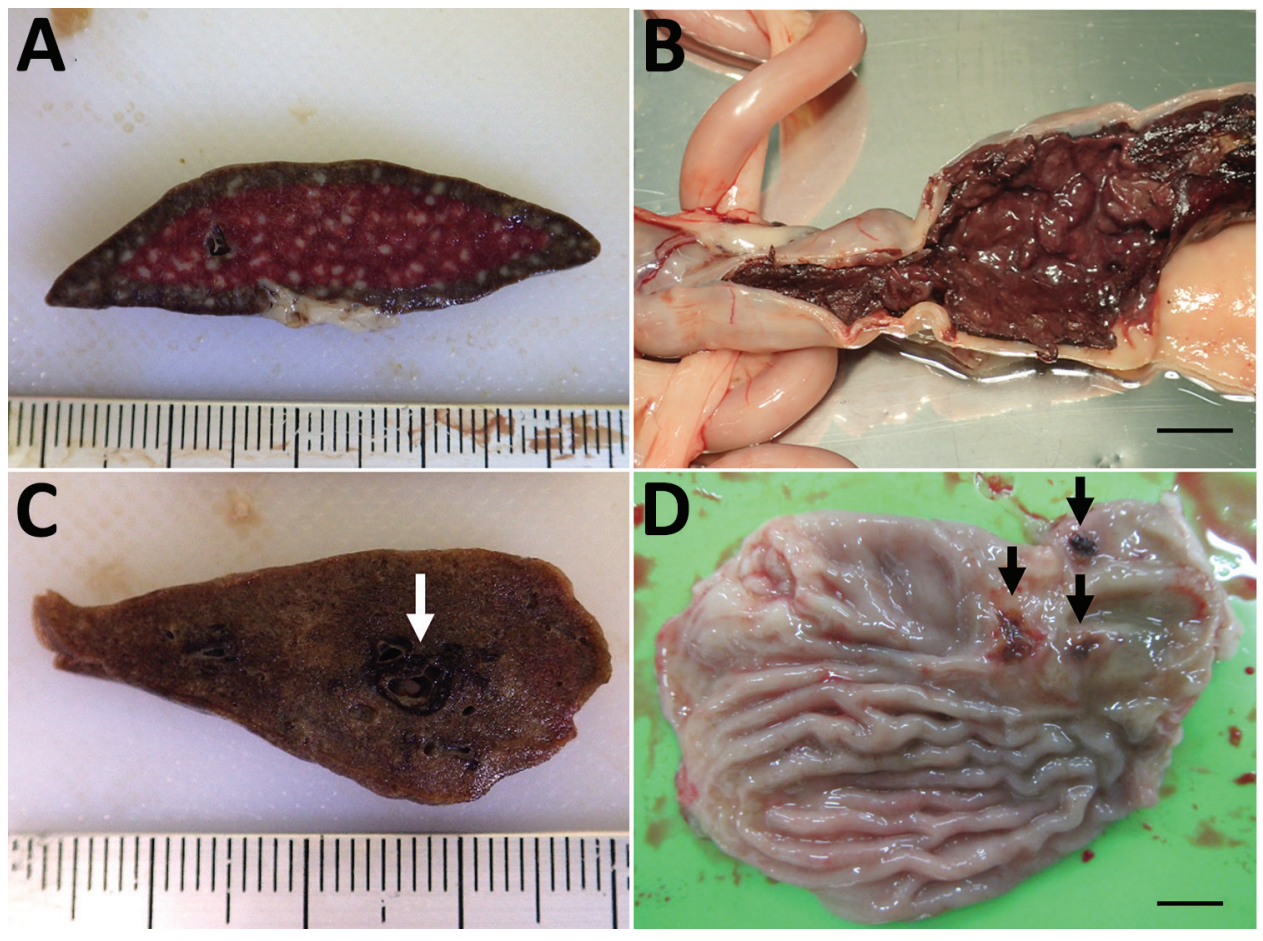

Figure 1. Gross pathology of lesions from cats with fatal severe fever with thrombocytopenia syndrome, Japan. A) Enlarged follicles (white spots) in the spleen. Ruler represents centimeters. B) Hemorrhage in the colon. Scale bar indicates $1 \mathrm{~cm}$. C) Hemorrhage in the lung; white arrow indicates pulmonary hemorrhage around the trachea. Ruler represents centimeters. D) Gastrointestinal ulcers (black arrows) were also seen in some cases. Scale bar indicates $1 \mathrm{~cm}$. 
Table 3. Lesions in the lymphatic system from 7 cats with fatal severe fever with thrombocytopenia syndrome, Japan*

\begin{tabular}{|c|c|c|c|c|c|c|c|}
\hline \multirow[b]{2}{*}{ Lesions } & \multicolumn{7}{|c|}{ Case no., $\mathrm{n}=$ no. lymph nodes assessed } \\
\hline & $1, n=4$ & $2, n=1$ & $3, n=4$ & $4, n=9$ & $5, n=7$ & $6, n=4$ & $7, n=6$ \\
\hline Hyperplasia without SFTSV-positive cells & 0 & 0 & 3 & 0 & 2 & 2 & 0 \\
\hline SFTSV-positive blastic lymphocytes & 3 & 1 & 1 & 6 & 2 & 1 & 2 \\
\hline Necrotizing lymphadenitis & 1 & 0 & 0 & 3 & 3 & 1 & 4 \\
\hline
\end{tabular}

structure in the intestinal submucosa; some of these cells infiltrated the lamina propria (Figure 3, panels A, B). We observed infiltration of these cells in all

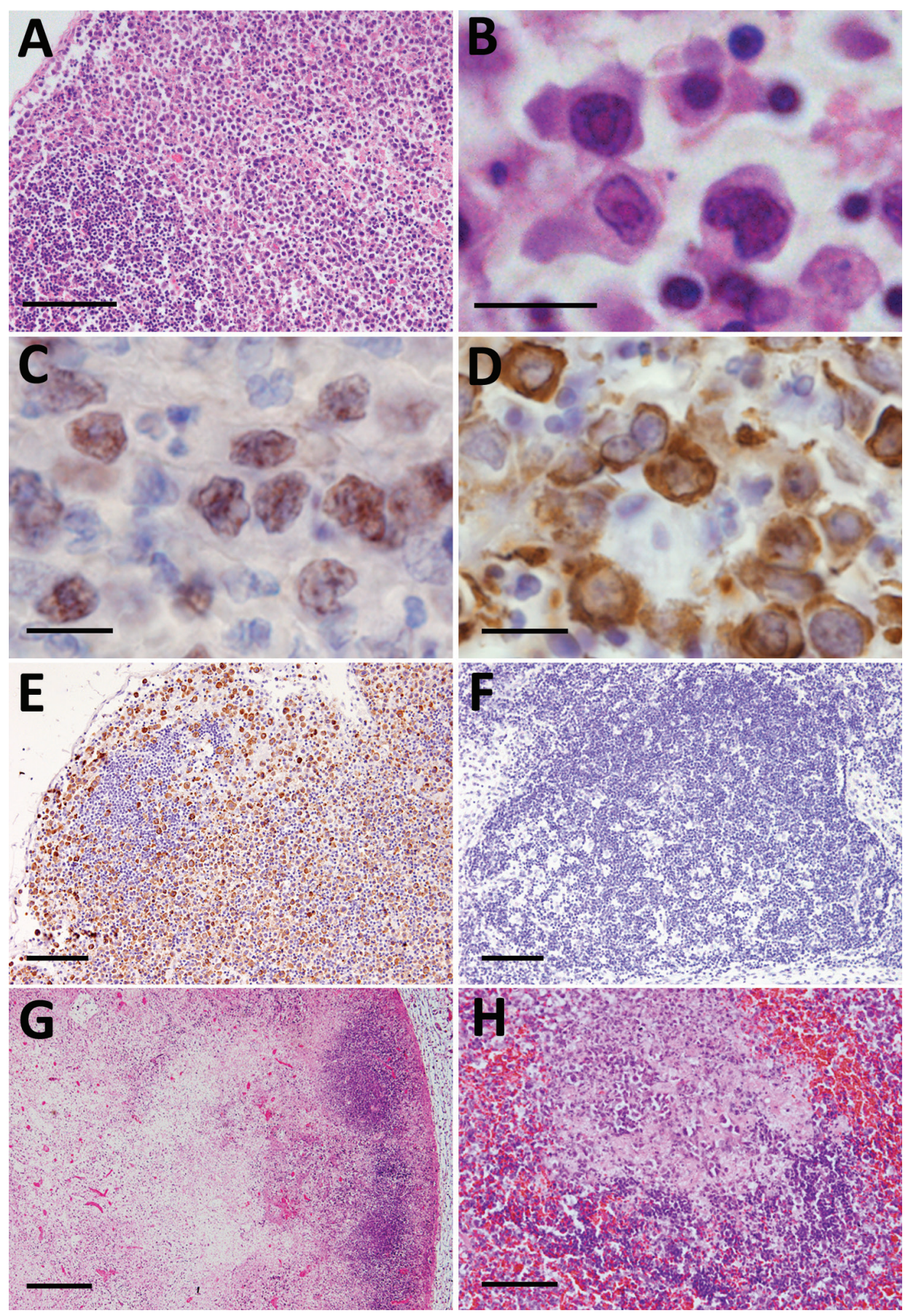

hemorrhage lesions and ulcers (Figure 3, panels C, D), suggesting a relationship between blastic lymphocytes and lesions.

Figure 2. Histopathological lesions in the lymphoid organs from fatal cases of severe fever with thrombocytopenia syndrome (SFTS) in cats, Japan. A) Hematoxylin \& eosin (HE)stained lymph node demonstrating accumulation of blastic

lymphocytes around the lymphoid follicle. Scale bar indicates $100 \mu \mathrm{m}$. B) HE-stained blastic lymphocytes from the lymph nodes demonstrating highly pleomorphic cells with large clear nuclei and prominent nucleoli, resembling immunoblasts. Scale bar indicates $10 \mu \mathrm{m} . \mathrm{C}, \mathrm{D})$ CD79a-stained (C) and immunohistochemistry-stained (D) blastic lymphocytes from the lymph nodes. Scale bar indicates $10 \mu \mathrm{m}$. E) Lymph node stained by immunohistochemistry revealing SFTS virus-positive blastic lymphocytes distributed around the follicle. Scale ar indicates 100 $\mu \mathrm{m}$. F) Immunohistochemistrystained hyperplastic lymph node demonstrating no SFTSV-positive cells or necrotic foci. Scale bar indicates $100 \mu \mathrm{m}$. G) Necrotic lymphadenitis in HE-stained lymph node. Scale bar indicates $200 \mu \mathrm{m}$. H) HE-stained spleen demonstrating necrotic lesions in the splenic follicle. Scale bar indicates $50 \mu \mathrm{m}$. 

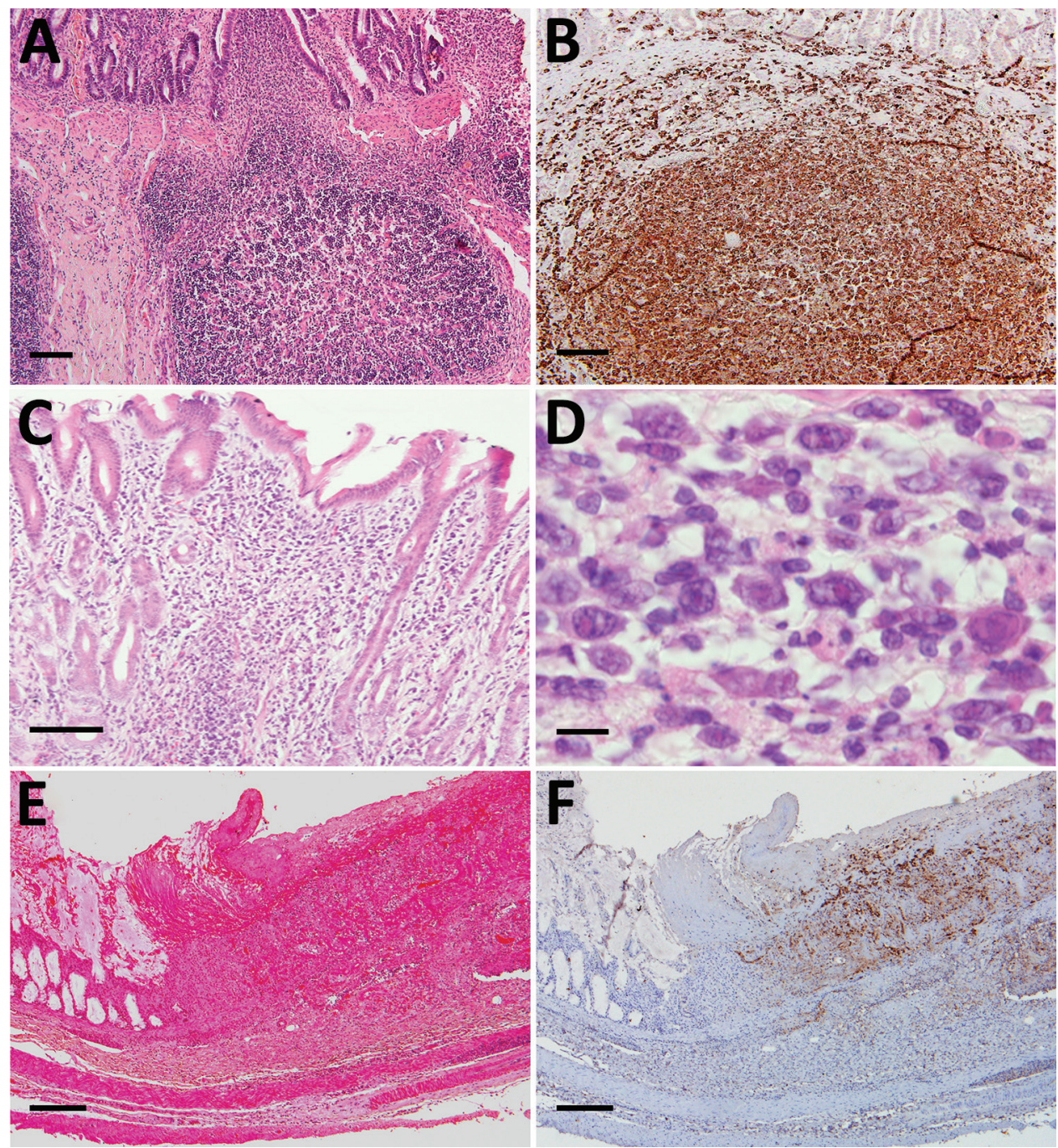

Figure 3. Histopathological lesions in the intestinal tracts from fatal cases of severe fever with thrombocytopenia syndrome (SFTS) in cats, Japan. A, B) Hematoxylin \& eosin (HE)-stained (A) and immunohistochemistry-stained (B) ileum sections demonstrating enlargement of Peyer's patch and accumulation of SFTSVpositive blastic lymphocytes. Scale bars indicates $100 \mu \mathrm{m}$. C) HE-stained colon sections demonstrating infiltration of lymphocytes into the lamina propria. Scale bar indicates 100 $\mu \mathrm{m}$. D) High power magnification of panel $\mathrm{C}$ demonstrating the infiltrating lymphocytes were blastic lymphocytes. Scale bar indicates $10 \mu \mathrm{m}$. E, F) HE stained (E) and immunohistochemistrystained $(F)$ ulcerative lesions in the cecum. Scale bars indicate $200 \mu \mathrm{m}$.

\section{Liver and Lung Lesions}

In the livers from 3 cats, we noted formation of small necrotic foci (Figure 4, panel A). The necrotic lesions always were surrounded by SFTSV-positive blastic lymphocytes (Figure 4, panel B), but the cells usually were distributed in the portal area. We also found bile pigmentation and hemophagocytic macrophages in the liver.

Feline SFTS pulmonary lesions consisted of hemorrhage in 3 cases and formation of necrotic foci in the interstitial tissues surrounding the trachea in 2 cases (Figure 4, panel C). Like hepatic necrotic foci, the pulmonary necrotic foci we observed in these cases also showed SFTSV-positive blastic lymphocytes (Figure 4, panel D).

\section{SFTSV-Positive Blastic Lymphocyte Characterization} Immunohistochemistry revealed that SFTSV-positive signals were limited to the blastic lymphocytes, and these were of B cell lineage and expressed CD79a (Figure 2, panel C). We performed further characterization by using immunohistochemical stains against Ig lambda chain and Ki67. The results demonstrated that SFTSV-positive atypical lymphocytes expressed Ig lambda chain and Ki67 (Figure 4, panels E, F). Hence, we considered these cells plasmablasts, which are immature plasma cells retaining proliferation activities.

The presence of SFTSV antigens in macrophages has been reported $(15,24)$. To investigate whether macrophages in our cases consisted of SFTSV-positive cells along with B cells, we performed double immunofluorescence analysis of the macrophage markers CD204 and SFTSV. The results demonstrated that SFTSV-positive cells were not CD204-positive (Figure 5).

\section{SFTSV-Positive Cells in the Thymus}

In addition to blastic lymphocytes, we found SFTSVpositive cells with spindle-to-polygonal morphology 


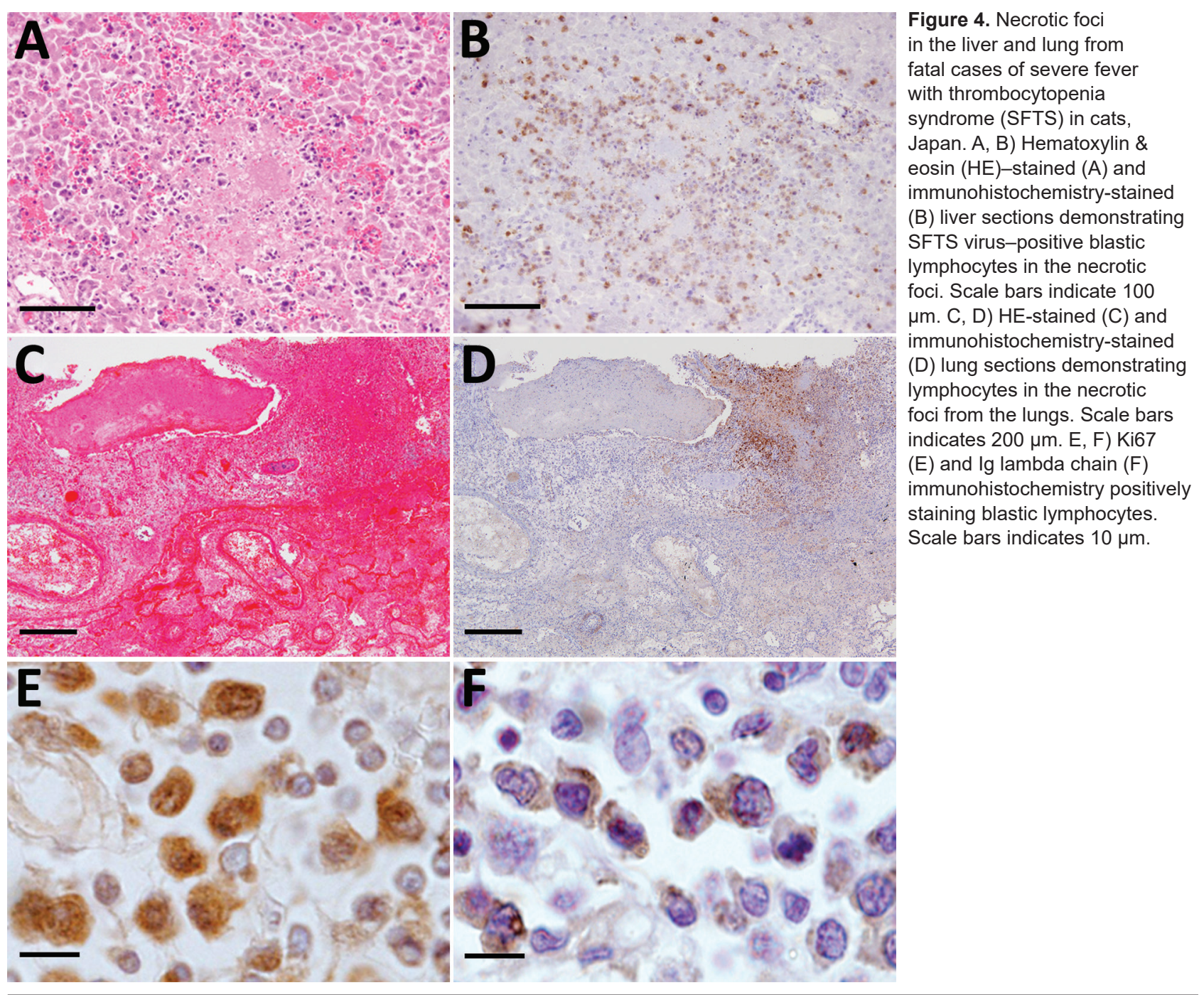

in the thymus gland. Double immunofluorescence analysis of SFTSV and cytokeratin revealed that these cells were thymic epithelial cells (Figure 6).

\section{Discussion}

We analyzed the pathological changes in 7 fatal cases of SFTS in domestic felids. We detected characteristic lesions in the lymphoid organs, including the lymph nodes and spleen. We observed gross enlargement and hemorrhage of multiple lymph nodes and formation of white spots in the spleen in all 7 cases (Figure 1 , panel D). Because these findings are highly suggestive of but not specific for feline SFTS, additional cases with these findings should be further examined to confirm viral infection by testing, such as RT-PCR.

Similar to human cases (20-22), we frequently observed necrotic lymphadenitis in this study and found accumulation of SFTSV-positive atypical lymphocytes in all 7 cases (Table 2). In addition, all 7 cats had necrotic splenitis, similar to human SFTS cases $(20,21)$. Therefore, lesions in the lymphoid organs, or accumulation of atypical B cells can be considered characteristic and highly specific for lesions in SFTS for both humans and felines. Furthermore, these lesions can be useful indicators of whether experimental infection in laboratory animals appropriately reproduces spontaneous SFTS. In fact, feline cases of experimental infection showed necrotic lymphadenitis and splenitis (13).

Although some reports have detected SFTSV antigens in macrophages, our study demonstrated that SFTSV-positive cells mostly were blastic lymphocytes (Figure 2, panels B-D; Figure 4, panels E, F). Immunofluorescence revealed that only punctate signals were detected in the macrophages (Figure 5). This result indicates that macrophages can phagocytose SFTSV but do not support SFTSV replication. Our findings also showed that SFTSV targets the cells of B-cell lineage 


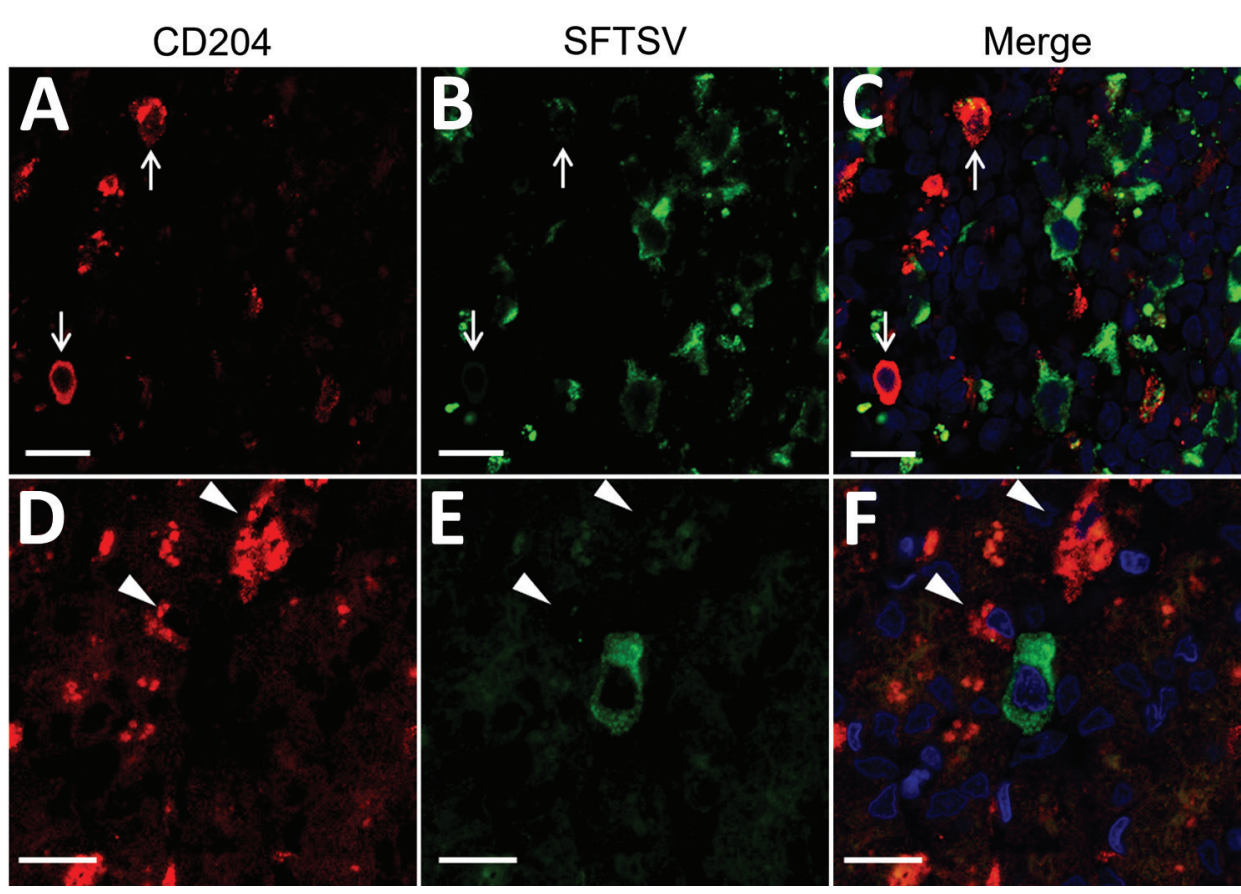

Figure 5. Double-labeling immunofluorescent staining of the lymph node $(A-C)$ and the liver (D-F) from fatal cases severe fever with thrombocytopenia syndrome (SFTS) in cats, Japan. Red indicates signals of CD204. Green indicates signals of SFTS virus. Blue indicates nuclei labeled with DAPI. Arrows in panels A-C indicate CD204-positive macrophages in the lymph node. Arrows in panels $D-F$ indicate CD204-positive kupffer cells in the liver. Scale bars indicate $10 \mu \mathrm{m}$.

and that plasmablasts were the predominant site of viral replication. Such tropism of SFTSV to plasmablast also has been reported in human cases and in vitro analysis clarified SFTSV targeted plasmablastic cell line, not B cell lymphoma cell lines (25). However, why large numbers of plasmablasts appeared and accumulated in the lymph nodes remains unclear. Dysregulated immunological response of plasmablasts to SFTSV infection and viral modulation of host-plasmablast dynamics are 2 possible causes of plasmablast accumulation in the lymph nodes. Furthermore, in all cases, we found SFTSV-positive blastic lymphocytes in and around the intestinal ulcerative lesions (Figure 3, panel F) and necrotic foci in the liver and lungs
(Figure 4, panels A-D), suggesting a role of blastic lymphocytes in the pathogenesis of ulcers and necrotic lesions. Depositing of Ig in the necrotic foci and expression of death ligands in SFTSV-positive cells should be analyzed in future cases. Expression of cell death-inducing factors on SFTSV-positive blastic lymphocytes, such as self-reactive Ig and death ligands, could indicate a relationship between these cells and necrotic foci; further study is warranted. Our study demonstrated that the thymic epithelial cells can be another target of SFTSV. However, the significance of viral infection in the thymic epithelium is unclear.

Gastrointestinal manifestation is one of the clinical features of human SFTS (1-4). A lethal case of
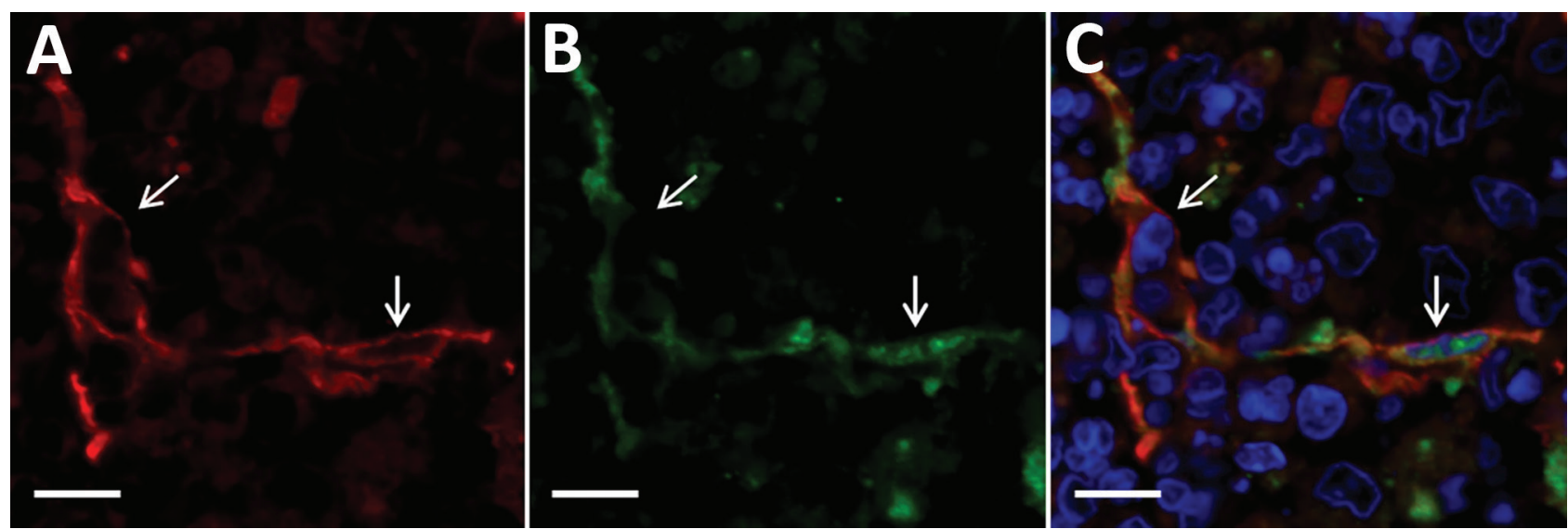

Figure 6. Double-labeling immunofluorescent staining of the thymus from fatal cases severe fever with thrombocytopenia syndrome (SFTS) in cats, Japan. Arrows indicate thymic epithelial cells. A, D) Red indicates signals of cytokeratin. B, E) Green indicates signals of SFTS virus. C, F) Blue indicates nuclei labeled with DAPI. Scale bars indicate $10 \mu \mathrm{m}$. 
severe intestinal hemorrhage and another case of multiple gastrointestinal ulceration have been reported in humans $(20,26)$. However, the incidence of these lesions is unclear because cases without obvious intestinal findings also have been reported (21). Experimental SFTSV infection in cats and our results have demonstrated a high rate of gastrointestinal hemorrhage (6/7 cases; Figure 1, panel A) and gastrointestinal ulcers (3/7 cases; Figure 3, panel E), which suggests that human and feline cases share a common pathogenesis and that feline cases show more severe gastrointestinal lesions than human cases (13). Pulmonary hemorrhage and necrotic foci also suggest the severity of feline SFTS. This severe pathogenesis in many tissues can cause high lethality, $\approx 70 \%$ (27), suggesting that feline SFTS is a typical lethal viral hemorrhagic fever.

Jaundice was frequently observed in our cases (Table 2) and in experimental infection in cats (13). Also, marked elevation of serum hepatic enzymes were detected in most cases (Table 1). However, in this study, morphologic lesions in the liver were only sporadic small necrotic foci, insufficient to cause systemic jaundice and marked elevation of serum hepatic enzymes. Microscopic lesions of the liver were also mild in human SFTS cases and in cats with experimental infection $(13,21)$. These findings suggest microscopically undetectable hepatic damage or functional failure of the hepatobiliary system. Further analysis is needed to clarify the mechanism of liver damage in SFTS.

Other typical clinical manifestations of SFTS in humans include neurologic signs, thrombocytopenia, and leukocytopenia (1-4). Although neurologic signs were unclear in the cats in our study, thrombocytopenia and leukocytopenia were clinically detected. Analysis of the bone marrow and central nervous system in feline SFTS cases will help clarify pathogenesis.

Our study demonstrated typical lesions of spontaneous fatal cases of feline SFTS, consisting of similar pathological lesions and a more severe hemorrhagic tendency than in human SFTS cases. Information on disease in animals that mimics human SFTS can help prevent animal-to-human transmission. Thus, we believe feline models can be used to study the pathogenesis of SFTS.

\section{Acknowledgments}

We thank Shigeru Morikawa for providing the antibody against SFTSV. We also thank Shiho Hamada and Mari Shimizu for supporting the necropsy and laboratory work.

This research was supported by Japan Agency for Medical Research and Development under grant no. $20 \mathrm{fk} 0108069$.

\section{About the Author}

Dr. Sakai is an assistant professor in the Laboratory of Veterinary Pathology, Joint Faculty of Veterinary Medicine, Yamaguchi University. His research interests include the histopathology and molecular pathology of animal infectious diseases, especially zoonotic viral diseases.

\section{References}

1. Kato H, Yamagishi T, Shimada T, Matsui T, Shimojima M, Saijo M, et al.; SFTS Epidemiological Research Group-Japan. Epidemiological and clinical features of severe fever with thrombocytopenia syndrome in Japan, 2013-2014. PLoS One. 2016;11:e0165207. https:// doi.org/10.1371/ journal.pone.0165207

2. Kim YR, Yun Y, Bae SG, Park D, Kim S, Lee JM, et al. Severe fever with thrombocytopenia syndrome virus infection, South Korea, 2010. Emerg Infect Dis. 2018;24:2103-5. https:// doi.org/10.3201/eid2411.170756

3. Li DX. Severe fever with thrombocytopenia syndrome: a newly discovered emerging infectious disease. Clin Microbiol Infect. 2015;21:614-20. https:// doi.org/10.1016/ j.cmi.2015.03.001

4. Saijo M. Pathophysiology of severe fever with thrombocytopenia syndrome and development of specific antiviral therapy. J Infect Chemother. 2018;24:773-81. https://doi.org/10.1016/j.jiac.2018.07.009

5. Yu XJ, Liang MF, Zhang SY, Liu Y, Li JD, Sun YL, et al. Fever with thrombocytopenia associated with a novel bunyavirus in China. N Engl J Med. 2011;364:1523-32. https://doi.org/10.1056/NEJMoa1010095

6. Robles NJC, Han HJ, Park SJ, Choi YK. Epidemiology of severe fever and thrombocytopenia syndrome virus infection and the need for therapeutics for the prevention. Clin Exp Vaccine Res. 2018;7:43-50. https:/ / doi.org/ 10.7774/cevr.2018.7.1.43

7. Luo LM, Zhao L, Wen HL, Zhang ZT, Liu JW, Fang LZ, et al. Haemaphysalis longicornis ticks as reservoir and vector of severe fever with thrombocytopenia syndrome virus in China. Emerg Infect Dis. 2015;21:1770-6. https:/ / doi.org/ 10.3201/eid2110.150126

8. Chen C, Li P, Li KF, Wang HL, Dai YX, Cheng X, et al. Animals as amplification hosts in the spread of severe fever with thrombocytopenia syndrome virus: A systematic review and meta-analysis. Int J Infect Dis. 2019;79:77-84. https://doi.org/10.1016/j.ijid.2018.11.017

9. Hwang J, Kang JG, Oh SS, Chae JB, Cho YK, Cho YS, et al. Molecular detection of severe fever with thrombocytopenia syndrome virus (SFTSV) in feral cats from Seoul, Korea. Ticks Tick Borne Dis. 2017;8:9-12. https:/ / doi.org/10.1016/ j.ttbdis.2016.08.005

10. Kimura T, Fukuma A, Shimojima M, Yamashita Y, Mizota F, Yamashita M, et al. Seroprevalence of severe fever with thrombocytopenia syndrome (SFTS) virus antibodies in humans and animals in Ehime prefecture, Japan, an endemic region of SFTS. J Infect Chemother. 2018;24:802-6.

https://doi.org/10.1016/j.jiac.2018.06.007

11. Matsuno K, Nonoue N, Noda A, Kasajima N, Noguchi K, Takano A, et al. Fatal tickborne phlebovirus infection in captive cheetahs, Japan. Emerg Infect Dis. 2018;24:1726-9. https:// doi.org/10.3201/eid2409.171667

12. Matsuu A, Momoi Y, Nishiguchi A, Noguchi K, Yabuki M, Hamakubo E, et al. Natural severe fever with thrombocytopenia syndrome virus infection in domestic cats 
in Japan. Vet Microbiol. 2019;236:108346. https:/ / doi.org/ 10.1016/j.vetmic.2019.06.019

13. Park ES, Shimojima M, Nagata N, Ami Y, Yoshikawa T, Iwata-Yoshikawa N, et al. Severe fever with thrombocytopenia syndrome phlebovirus causes lethal viral hemorrhagic fever in cats. Sci Rep. 2019;9:11990. https:/ / doi.org/10.1038/s41598-019-48317-8

14. Kida K, Matsuoka Y, Shimoda T, Matsuoka H, Yamada H, Saito T, et al. A case of cat-to-human transmission of severe fever with thrombocytopenia syndrome virus. Jpn J Infect Dis. 2019;72:356-8. https:/ / doi.org/10.7883/ yoken.JJID.2018.526

15. Jin C, Liang M, Ning J, Gu W, Jiang H, Wu W, et al. Pathogenesis of emerging severe fever with thrombocytopenia syndrome virus in C57/BL6 mouse model. Proc Natl Acad Sci U S A. 2012;109:10053-8. https:/ / doi.org/10.1073/pnas.1120246109

16. Liu Y, Wu B, Paessler S, Walker DH, Tesh RB, Yu XJ. The pathogenesis of severe fever with thrombocytopenia syndrome virus infection in alpha/beta interferon knockout mice: insights into the pathologic mechanisms of a new viral hemorrhagic fever. J Virol. 2014;88:1781-6. https:/ / doi.org/10.1128/JVI.02277-13

17. Tani H, Fukuma A, Fukushi S, Taniguchi S, Yoshikawa T, Iwata-Yoshikawa N, et al. Efficacy of T-705 (favipiravir) in the treatment of infections with lethal severe fever with thrombocytopenia syndrome virus. MSphere. 2016;1: e00061-15. https://doi.org/10.1128/mSphere.00061-15

18. Jin C, Jiang H, Liang M, Han Y, Gu W, Zhang F, et al. SFTS virus infection in nonhuman primates. J Infect Dis. 2015;211:915-25. https:// doi.org/10.1093/infdis/jiu564

19. Gowen BB, Westover JB, Miao J, Van Wettere AJ, Rigas JD, Hickerson BT, et al. Modeling severe fever with thrombocytopenia syndrome virus infection in golden Syrian hamsters: importance of STAT2 in preventing disease and effective treatment with favipiravir. J Virol. 2017;91: e01942-16. https:// doi.org/10.1128/JVI.01942-16

20. Uehara N, Yano T, Ishihara A, Saijou M, Suzuki T. Fatal severe fever with thrombocytopenia syndrome: an autopsy case report. Intern Med. 2016;55:831-8. https:// doi.org/10.2169/internalmedicine.55.5262
21. Hiraki T, Yoshimitsu M, Suzuki T, Goto Y, Higashi M, Yokoyama S, et al. Two autopsy cases of severe fever with thrombocytopenia syndrome (SFTS) in Japan: a pathognomonic histological feature and unique complication of SFTS. Pathol Int. 2014;64:569-75. https://doi.org/10.1111/pin.12207

22. Takahashi T, Maeda K, Suzuki T, Ishido A, Shigeoka T, Tominaga $\mathrm{T}$, et al. The first identification and retrospective study of severe fever with thrombocytopenia syndrome in Japan. J Infect Dis. 2014;209:816-27. https:/ / doi.org/10.1093/ infdis/jit603

23. Yoshikawa T, Fukushi S, Tani H, Fukuma A, Taniguchi S, Toda S, et al. Sensitive and specific PCR systems for detection of both Chinese and Japanese severe fever with thrombocytopenia syndrome virus strains and prediction of patient survival based on viral load. J Clin Microbiol. 2014;52:3325-33. https:// doi.org/10.1128/JCM.00742-14

24. Nakano A, Ogawa H, Nakanishi Y, Fujita H, Mahara F, Shiogama K, et al. Hemophagocytic lymphohistiocytosis in a fatal case of severe fever with thrombocytopenia syndrome. Intern Med. 2017;56:1597-602. https://doi.org/10.2169/ internalmedicine.56.6904

25. Suzuki T, Sato Y, Sano K, Arashiro T, Katano H, Nakajima N, et al. Severe fever with thrombocytopenia syndrome virus targets B cells in lethal human infections. J Clin Invest. 2020;130:799-812. https:/ / doi.org/10.1172/JCI129171

26. Kaneyuki S, Yoshikawa T, Tani H, Fukushi S, Taniguchi S, Fukuma A, et al. Ulcerative lesions with hemorrhage in a patient with severe fever with thrombocytopenia syndrome observed via upper gastrointestinal endoscopy. Jpn J Infect Dis. 2016;69:525-7. https:/ / doi.org/10.7883/yoken.JJID.2015.404

27. Maeda K, Ishijima K, Tatemoto K, Sakai Y, Shimoda H, Takano A, et al. Animals that develop SFTS (mainly dogs and cats) [In Japanese]. Infect Agents Surveill Rep. 2019;40:118-9. https://www.niid.go.jp/niid/ja/allarticles/surveillance/ 2467-iasr/related-articles/related-articles-473/8988-473r06.html

Address for correspondence: Yusuke Sakai, Laboratory of Veterinary Pathology, Joint Faculty of Veterinary Medicine, Yamaguchi University, 1677-1 Yoshida, Yamaguchi-shi, Yamaguchi 753-8515, Japan; email: vsakai@yamaguchi-u.ac.jp 